\title{
Unique Mapping of Structural and Functional Connectivity on Cognition
}

\author{
ㄱoelle Zimmermann, John D. Griffiths, and @Anthony R. McIntosh \\ Baycrest Health Sciences, Rotman Research Institute, Toronto, Ontario M6A 2E1, Canada
}

The unique mapping of structural brain connectivity (SC) and functional brain connectivity (FC) on cognition is currently not well understood. It is not clear whether cognition is mapped via a global connectome pattern or instead is underpinned by several sets of distributed connectivity patterns. Moreover, we also do not know whether the spatial distributions of SC and FC that underlie cognition are overlapping or distinct. Here, we study the relationship between SC and FC and an array of psychological tasks in 609 subjects (males, 269; females, 340) from the Human Connectome Project. We identified several sets of connections that each uniquely map onto cognitive function. We found a small number of distributed SCs and a larger set of corticocortical and corticosubcortical FCs that express this association. Importantly, the SC and FC each show unique and distinct patterns of variance across subjects as they relate to cognition. The results suggest that a complete understanding of connectome underpinnings of cognition calls for a combination of the two modalities.

Key words: behavior; cognition; functional connectivity; individual; structural connectivity

\section{Significance Statement}

Structural connectivity (SC), the physical white-matter inter-regional pathways in the brain, and functional connectivity (FC), the temporal coactivations between the activity of the brain regions, have each been studied extensively. Little is known, however, about the distribution of variance in connections as they relate to cognition. Here, in a large sample of subjects $(N=609)$, we showed that two sets of brain- behavior patterns capture the correlations between SC and FC with a wide range of cognitive tasks, respectively. These brain-behavior patterns reveal distinct sets of connections within the SC and the FC network and provide new evidence that $\mathrm{SC}$ and FC each provide unique information for cognition.

\section{Introduction}

In neuroscience, big data initiatives such as the Human Connectome Project (HCP) acquire connectomic and phenotypic data from a large number of individuals in an effort to understand how brain networks relate to individual behavior (Van Essen et al., 2013; Fornito et al., 2016). Connectomes can represent either structural connectivity (SC), the white matter inter-regional pathways estimated from diffusion-weighted MRI (dwMRI; Baldassarre et al., 2012), or functional connectivity (FC), the patterns of temporal dependencies between regional activity mea-

Received April 5, 2018; revised Sept. 4, 2018; accepted Sept. 8, 2018.

Author contributions: J.Z. designed research;J.Z. performed research;J.D.G. and A.R.M. contributed unpublished reagents/analytic tools; J.Z. analyzed data; J.Z. wrote the paper.

Data were provided by the Human Connectome Project, WU-Minn Consortium (Principal Investigators: David Van Essen and Kamil Ugurbil; Grant 1U54MH091657) funded by the 16 National Institutes of Health (NIH) institutes and centers that support the NIH Blueprint for Neuroscience Research; and by the McDonnell Center for Systems Neuroscience at Washington University. The research was also supported Natural Sciences and Engineering Research Council of Canada Grant RGPIN-2017-06793.

The authors declare no competing financial interests.

Correspondence should be addressed to Joelle Zimmermann, Baycrest Health Sciences, Rotman Research Institute, 3560 Bathurst Street, Toronto, 0N M6A 2E1, Canada. E-mail: jzimmermann@research.baycrest.org.

https://doi.org/10.1523/JNEUROSCI.0900-18.2018

Copyright $\odot 2018$ the authors $\quad 0270-6474 / 18 / 389658-10 \$ 15.00 / 0$ surements such as blood oxygenation level-dependent (BOLD) functional magnetic resonance imaging (fMRI) time series. The most commonly studied form of FC, resting-state FC (rsFC), is measured in the absence of an explicit task. It represents meaningful coordinated fluctuations that have been related to task FC and performance (Mennes et al., 2010; Baldassarre et al., 2012; Stevens and Spreng, 2014).

Both SC (Matejko et al., 2013; Willmes et al., 2014; Moeller et al., 2015; Klein et al., 2016) and rsFC (Song et al., 2008, 2009; Pamplona et al., 2015; Santarnecchi et al., 2015; Hearne et al., 2016; Smith, 2016; Pezoulas et al., 2017; Ferguson et al., 2017) have been linked to cognitive functioning, including higher-order cognitive processes such as fluid and crystallized intelligence, visuospatial processing (Ponsoda et al., 2017), or numerical cognition (Matejko et al., 2013; Willmes et al., 2014; Moeller et al., 2015; Klein et al., 2016).

What remains unclear, however, is the specificity of the behaviorally relevant aspect of the connectome. That is, can individual variability across connectomes be explained by a single set of connections that is highly predictive of cognitive function more generally? Or are there multiple sets of connections that each predict different aspects of cognitive functioning? The for- 
Table 1. Cognitive tests and corresponding function

\begin{tabular}{llc}
\hline Test & Function & Mean (SD), range \\
\hline ListSort_AgeAdj & Working memory (list sorting) & 103 (13), 63-141 \\
PicSeq_AgeAdj & Episodic memory (picture sequence memory) & 106 (17), 56-135 \\
CardSort_AgeAdj & Executive function/cognitive flexibility (dimensional change card sort) & $102(10), 58-123$ \\
Flanker_AgeAdj & Executive function/inhibition (flanker task) & $102(10), 74-124$ \\
ProcSpeed_AgeAdj & Processing speed (pattern completion processing speed) & $103(19), 45-149$ \\
WM_Task_2bk_Acc & Working memory accuracy (2-back task) & $83(11), 37-100$ \\
WM_Task_2bk_Median_RT & Working memory reaction time (2-back task) & $970(143), 602-1440$ \\
WM_Task_obk_Acc & Working memory accuracy (0-back task) & $89(11), 48-100$ \\
WM_Task_Obk_Median_RT & Working memory reaction time (0-back task) & $775(139), 506-1571$ \\
PMAT24_A_CR & Fluid intelligence correct responses (Penn progressive matrices) & $17(5), 5-24$ \\
PMAT24__RTCR & Fluid intelligence reaction time, correct responses (Penn progressive matrices) & $15,454(2976), 1989-61,641$ \\
\hline
\end{tabular}

mer suggests that connectomes are relevant for understanding general differences in global cognitive functioning, while the latter suggests that individual differences in particular networks can inform specific types of cognitive functioning, such as attention, memory, or executive function. Evidence of both views exists. For instance, Rosenberg et al. (2013) showed that a particular set of connectivity patterns predicted individuals' attention ability, supporting the notion of specific connectome-behavior associations. The connections identified were specific to attention ability and did not predict cognition more generally. In contrast, other research has shown that a single mode of covariation can capture relationships between a distributed set of functional connections and a wide set of behavioral and demographic variables (Smith et al., 2015). The same question can be asked for SC.

Currently, little is known about the overlap between the two modalities in their mapping to individual differences in cognition. Given the limitations of mapping individual SC to FC (Koch et al., 2002; Skudlarski et al., 2008; Honey et al., 2009; Zimmermann et al., 2016, 2018a,b), we might expect that the two modalities provide unique and distinct sources of cognitive-related variability (Duda et al., 2010; Hirsiger et al., 2016). On the other hand, cognition arises from an interplay of structure and function, and so a degree of overlap in the spatial pattern of networks that give rise to cognitive function is expected.

In the present study, we examine how cortical and subcortical SC and rsFC from 609 subjects from the Human Connectome Project relates to a wide range of cognitive functions, including working memory (WM), executive function/cognitive flexibility, processing speed, fluid intelligence, episodic memory, and attention/inhibitory control. We examined (1) whether there exists a single set of connections that generally map onto cognition, or rather several sets of connections that map onto cognition; and (2) whether the patterns of connectivity that map onto cognition are independent and unique for SC versus rsFC or whether they provide common information. We used partial least squares (PLS) to map orthogonal patterns of brain-behavior relationships (McIntosh and Lobaugh, 2004; Krishnan et al., 2011).

\section{Materials and Methods \\ Subjects and behavioral measures}

The sample included 609 genetically unrelated subjects (males, 269; females, 340; age range, 22-36 years) from the Q7 HCP release (MGH-USC Human Connectome Project; RRID:SCR_003490; Van Essen et al., 2013). The research was performed in compliance with the Code of Ethics of the World Medical Association (Declaration of Helsinki). All subjects provided written informed consent, and procedures were approved by the ethics committee in accordance with guidelines of WU-Minn HCP. In the current study, 11 behavioral measures of cognitive function were correlated with SC and FC (described below). Cognitive measures covered a range of processes. Note that the working memory 0 -back and 2-back tasks each included the following two measures: accuracy and reaction time. The measures are described in Table 1 and in detail in the spreadsheet version of the Data Dictionary available here: https://wiki. humanconnectome.org/display/PublicData/HCP + Data + Dictionary+ Public- + Updated + for + the $+1200+$ Subject + Release. Note that cognitive scores were age adjusted.

\section{MRI acquisition and preprocessing}

Data acquisition details for the WU-Minn HCP corpus (Van Essen et al., 2013) were described in detail previously (Smith et al., 2013; Sotiropoulos et al., 2013; Uğurbil et al., 2013). The present study made use of restingstate BOLD fMRI, multishell (multiple nonzero $b$-values) high-resolution dwMRI, and structural (T1-weighted MRI) data from the HCP minimally preprocessed pipeline (Glasser et al., 2013; Smith et al., 2013).

Diffusion data with a spin-echo multiband EPI sequence, with 111 slices of $1.25 \mathrm{~mm}$ isotropic voxels (Feinberg et al., 2010; Sotiropoulos et al., 2013; Uğurbil et al., 2013) and distortion corrected (Andersson et al., 2003; Andersson and Sotiropoulos, 2015), were used. Segmentation and parcellation were performed on the basis of the high-resolution T1weighted image (voxel size, $0.7 \mathrm{~mm}$ isotropic) of each subject using FreeSurfer (Fischl, 2012), automatically parcellating the brain into 83 cortical and subcortical ROIs (41 per hemisphere, plus brainstem) according to the Lausanne 2008 atlas (Daducci et al., 2012). An $83 \times 83$ connectivity matrix was formed, representing their reconstructed pathways for each pair of regions. Deterministic tractography using Dipy software (Garyfallidis et al., 2014) was performed on minimally preprocessed data using a method similar to that of Hagmann et al. (2008). Streamlines were computed from 60 equally spaced points within each voxel on the gray matter-white matter interface via the EuDX algorithm (Garyfallidis, 2012). Streamlines that were shorter than $10 \mathrm{~mm}$ or longer than $250 \mathrm{~mm}$ were discarded, as well as those that did not terminate at the gray matterwhite matter interface. The strength of the reconstructed connections was measured as streamline density, which was computed as the number of tractography streamlines that touched both cortical regions (Hagmann et al., 2008).

Whole brain echoplanar images ( $\mathrm{TR}=720 \mathrm{~ms} ; 2 \mathrm{~mm}^{3}$ voxels; Moeller et al., 2010; Uğurbil et al., 2013) with denoising procedures from the resting-state FIX (FMRIB ICA-based X-noiseifier) denoised dataset were used (Glasser et al., 2013; Smith et al., 2013). Data included both phaseencoding acquisition directions [left (L)-right (R), R-L; acquisition time, $14 \mathrm{~min}$ and $33 \mathrm{~s}$ ). We computed average regional time series from these voxels, based on the 83 cortical and subcortical regions. Pearson's correlations were calculated for each pair of time series to compute the FC between all regions; these were then Fisher $z$-transformed, and the two pairs of matrices ( $\mathrm{L}-\mathrm{R}$ and $\mathrm{R}-\mathrm{L}$ ) were averaged for each subject. The resulting FC matrices were $83 \times 83$ for the 609 subjects. Global signal regression was not performed for comparability with previous resting-state studies. Note that age was regressed from SC and Fisher's $z$-transformed FC, and residuals were used for analysis.

\section{Experimental design and statistical analysis}

Cognitive measures. The 11 cognitive measures were correlated (Pearson's) with one another to quantify the relationship between them. The 
resulting $p$ value cross-correlation matrix was of size $11 \times 11$. The $p$ value of each correlation value was corrected for multiple comparisons using false discovery rate (FDR), MATLAB function fdr_bky (MATLAB; RRID:SCR_001622; Benjamini et al., 2006). We also wanted to understand whether the cognitive measures describe a single global cognitive component of intelligence or whether numerous components are required to capture the different subtypes of cognitive functioning. To this end, we conducted a principal components analysis (PCA) of the cognitive measures (MATLAB function princomp). To assess the significance of the resulting eigenvalues, we permuted the cognitive measures 100 times (scrambled across cognitive measures and subjects) and decomposed (PCA) the permuted cognitive matrices to obtain a null distribution of eigenvalues for each principal component (PC). We then calculated the $p$ value per PC eigenvalue as the proportion of times the permuted eigenvalue exceeded the obtained eigenvalue.

Correlation between cognition and connectivity. We used PLS for neuroimaging (McIntosh and Lobaugh, 2004; Krishnan et al., 2011) to assess the multidimensional connectome-cognition relationships (behavioral PLS correlation, custom MATLAB code). As the connectivity matrices are symmetric, we used the vectorized upper triangle of the connectivity matrices from the 609 subjects. Structural connections that were 0 for $66 \%$ of subjects or more were not included in the analysis, resulting in 870 remaining connections for SC. The resulting input matrix to PLS consisted of $25 \%$ of zeros across all subjects and connections removed connections in the SC were randomly distributed throughout the network. Note that zero connections are not an issue for PLS per se but rather for resampling statistics, which can produce low variance as a result. The entire vectorized upper triangle was included for the FC, which included 3403 connections. Vectorized connectomes were then stacked, resulting in a subjects ${ }^{\star}$ connections brain matrix, for $\mathrm{SC}=609^{\star} 870$, and for $\mathrm{FC}=609^{\star} 3403$. The behavioral matrix was subjects ${ }^{\star}$ behavioral measures, of size $609^{\star} 11$.

The brain and behavioral matrices were then cross-correlated to start the PLS analysis. PLS is a multivariate analysis method comparable to canonical correlation which, however, optimizes covariance rather than correlations and captures the relationship between input matrices within mutually orthogonal latent variables (LVs). We calculated the correlations between input cross-correlation brain, and behavioral input matrices. The significance of LVs is assessed via permutation tests (1000 iterations) of the singular values from singular value decomposition (SVD) of the brain and behavioral correlation matrices, and the reliability of each connectivity estimate to the LV is assessed via bootstrap resampling (3000 iterations). The reliability of the loading of each connection onto the brain-behavior relationship in each LV is represented as a bootstrap ratio, the ratio of the weight of a connection over its estimated SE. The ratio can be considered equivalent to a $z$-score, but is used to impart reliability rather than significance. A connection with a positive high bootstrap ratio contributes positively and reliably to the brain-behavior correlation obtained for that $\mathrm{LV}$, whereas a connection with a negative high bootstrap ratio contributes negatively and reliably to the brain-behavior relationship. Bootstrapping is also used to construct confidence intervals on the brain-behavior correlations.

SC-cognition versus FC-cognition. To compare the connections that contributed to the SC-cognition relationship and those that contributed to the FC-cognition relationship, we calculated the scalar dot product (MATLAB function dot) between the brain scores $(U)$ from the PLS SVD expressed in the FC-cognition relationship and those expressed in the SC-cognition relationship, across all significant LVs. To compare the behavioral patterns that contributed to the SC-cognition and the FCcognition relationships, we calculated the dot product between the behavioral scores $(V)$ from the SC-cognition and FC-cognition SVD. To show whether highly behaviorally performing individuals identified by the SC-cognition analysis were the same as those identified by the FCcognition analysis, we correlated (Pearson's) individual subject behavioral scores between SC-cognition and FC-cognition LVs. Note that correlations were corrected for multiple comparisons using FDR (MATLAB function fdr_bky; Benjamini, 2006). Last, to assess how cognitive PCs mapped onto connectivity-cognition latent variables, we calculated the dot product between PCA scores and latent variable correlations (Zimmermann et al., 2018a).

\section{Results}

\section{Cognitive measures}

The cognitive scores correlated positively among one another, with the exception of the WM reaction time tasks, which correlated negatively with all other cognitive tests (Fig. 1). All correlations were significant (all $p<0.001$, multiple comparison corrected; see Materials and Methods).

A PCA of the 11 cognitive test scores yielded three significant PCs, where significance was assessed by permutation testing (see Materials and Methods; $\lambda=3.5072,1.8679$, and 1.2026; percentage of variance explained $=61 \%, 17 \%, 7 \%$, respectively; Fig. 2 ). All cognitive tests loaded onto the first PC, with negative WM reaction time loadings representing a processing speed component. Thus, participants who were more accurate (positive accuracy loading) were also faster (negative reaction time loading) on these WM tests. The second PC emphasized the Penn progressive matrices, tests of fluid intelligence (PMAT) tests; note that the reaction time (RT) for correct responses and the number of correct responses were positively correlated. The third PC emphasized similarities between the two WM RT tests and the remaining tests, in opposition to the two WM accuracy measures.

\section{Correlation between cognition and connectivity}

PLS analyses identified two significant LVs that describe the relationship between SC and cognition, and FC and cognition each, respectively.

\section{SC-cognition}

The two LVs that captured the SC-cognition association revealed two distinct patterns of cognitive functions that mapped onto two sets of unique structural connections (Fig. 3; LV1 SC: 37.22\% of total covariance; singular value $=2.53 ; p=0.04$; LV2 SC: $14.81 \%$ of total covariance; singular value $=1.6 ; p=0.03$ ). For both LVs, a small number of connections across cortical and subcortical regions was stable by bootstrap. Connections loaded positively and negatively onto each of the SC-cognition LVs (Fig. $3 B, D$, bootstrap ratios). For the cognitive measures, the first $\mathrm{LV}$ strongly expressed the full array of cognitive tests, while the second LV expressed primarily the two PMAT tests.

\section{FC-cognition}

The two LVs that captured the FC-cognition association revealed two distinct patterns of cognitive functions that map onto two sets of functional connections (Fig. 4; LV1 FC: $41.5 \%$ of total covariance; singular value $=6.45 ; p=0.01 ; \mathrm{LV} 2 \mathrm{FC}$ : $33 \%$ of total covariance; singular value $=5.75 ; p=0.046$ ).

LV1 revealed $\mathrm{FC}-$ cognition correlations expressed across an array of cognitive tests, and a large number of cortical, mainly frontoparietal, connections (as well as insula, transverse temporal) that load negatively onto this relationship (Fig. $4 B, D$, bootstrap ratios). Thus, better performance on card sorting, processing speed, WM accuracy, as well as the three RT measures (better performance $=$ lower RT) was associated with lower FC. Only four connections loaded negatively onto this LV, three of which were connections of the R entorhinal. LV2 revealed FCcognition correlations, expressed primarily with the PMAT tests. A large number of interhemispheric corticocortical and corticosubcortical connections loaded positively onto this LV. Thus, better performance on a PMAT test correlated with higher FC.

\section{SC-cognition versus FC-cognition}

As evident from Figures 3 and 4, there were more connections in the FC that correlate with cognition compared with the SC. This 


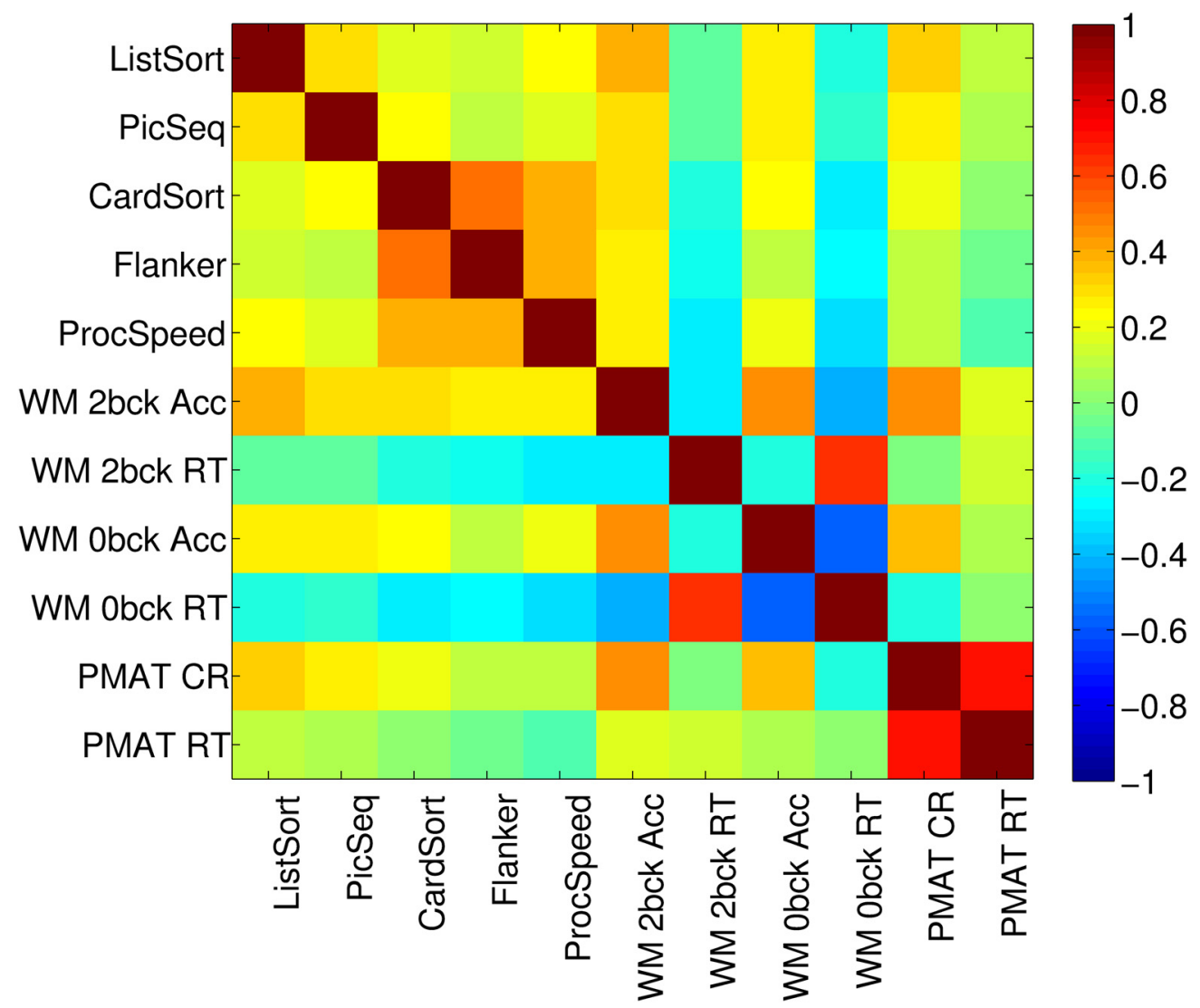

Figure 1. Correlation (Pearson's) among the cognitive tests.

A

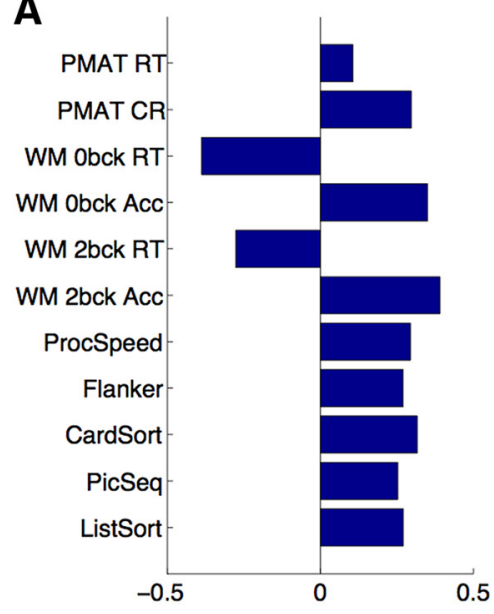

B

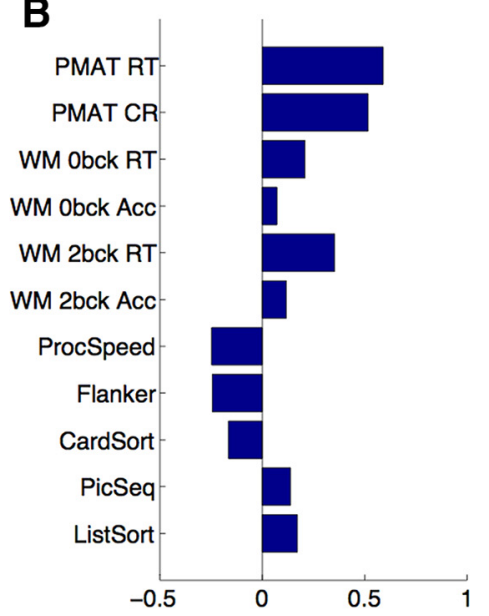

C

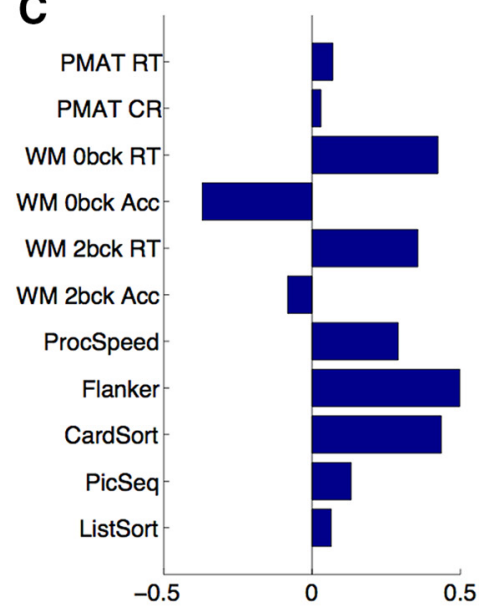

Figure 2. PCA of the 11 cognitive tests. Shown here are the principal component coefficients (loadings) on each PC. A, PC1. B, PC2.C, PC3.

can be shown as the number of connections that exceeded the bootstrap ratio threshold for each connection-cognition LV, expressed as a proportion of the number of connections that entered the PLS analysis (FC, 3403; SC, 870). The proportion of connections that exceeded the bootstrap threshold were as follows: FC: $L V 1=15 \%$; LV2 = 9\%; SC: LV1 = 2\%; LV2 = 1\%. We hypothesized that this may be due to the difference in total between-subjects variance in the SC and FC. To this end, we conducted an SVD of the subjectwise SC $(609$ subjects $\times 870$ connections) as well as the subjectwise FC (609 subjects $\times 3403$ connections) and compared the sum of the squared singular values corrected by the number of connections that entered the analysis, to account for the sparsity of the SC $\left(\Sigma S_{\text {corrected }}^{2}\right)$. Indeed, we found that the total corrected variance in subjectwise SC was smaller than that in the subjectwise FC $\left(\Sigma S_{\text {SC_corrected }}^{2}=\right.$ $0.0198 ; \Sigma S_{\text {FC_corrected }}^{2}=0.0294$ ).

We observed that a very different pattern of connections in the SC related to cognition than in the FC. This can be expressed quantitatively as the dot product of the PLS brain scores $(U)$ from the FC-cognition SVD and from the SC-cognition SVD, across both LVs. These dot products were close to zero (Table 2), suggesting that brain connectivity patterns show little overlap across modalities. This comparison was made for the behavior contributions to each LV as well. To this end, we calculated the dot 
SC LV1

Frontal
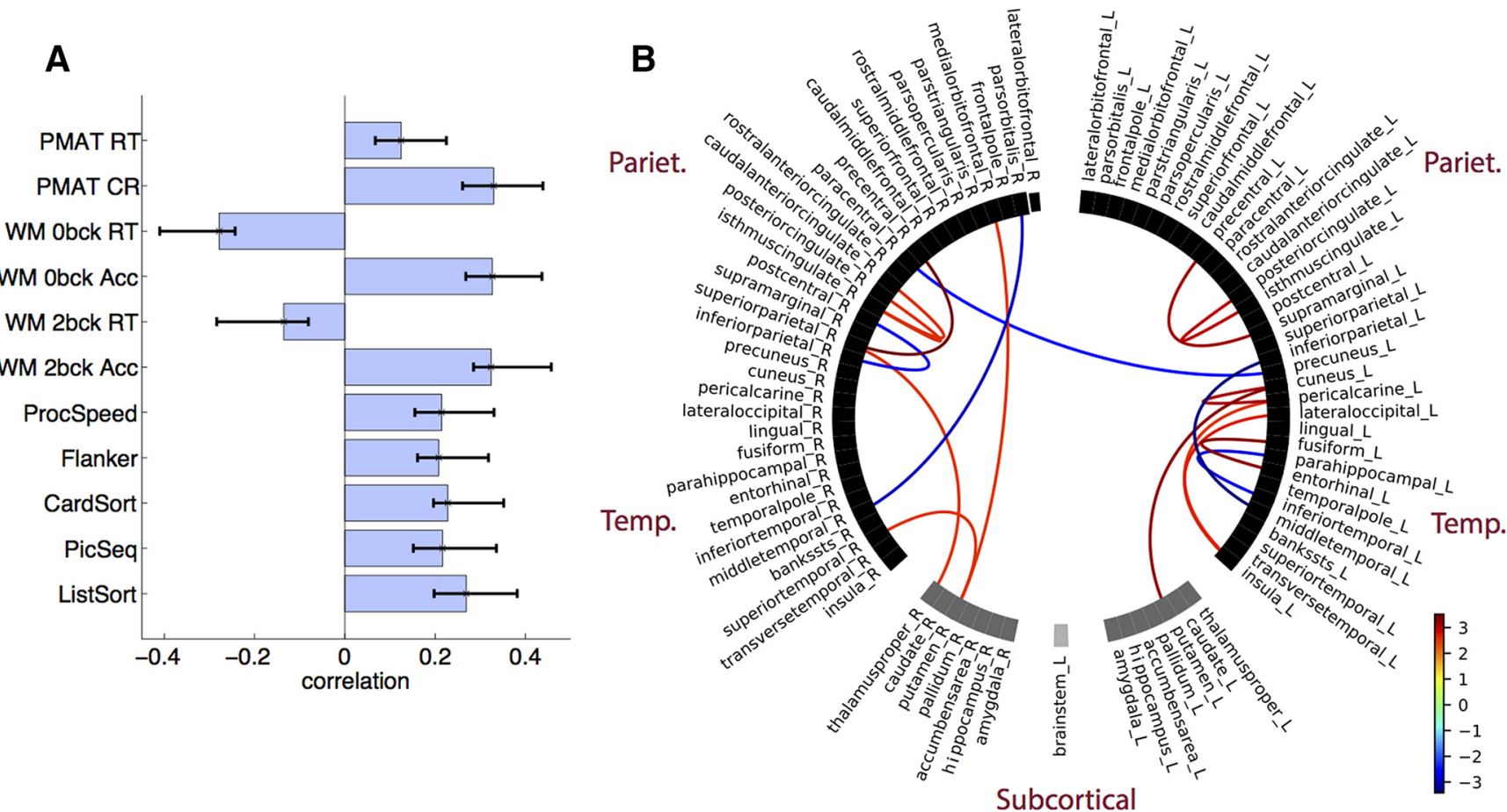

SC LV2

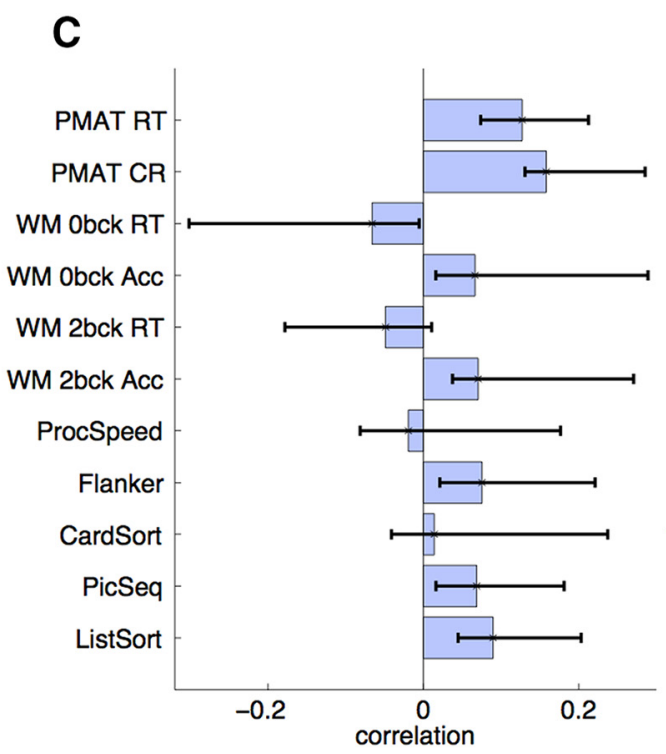

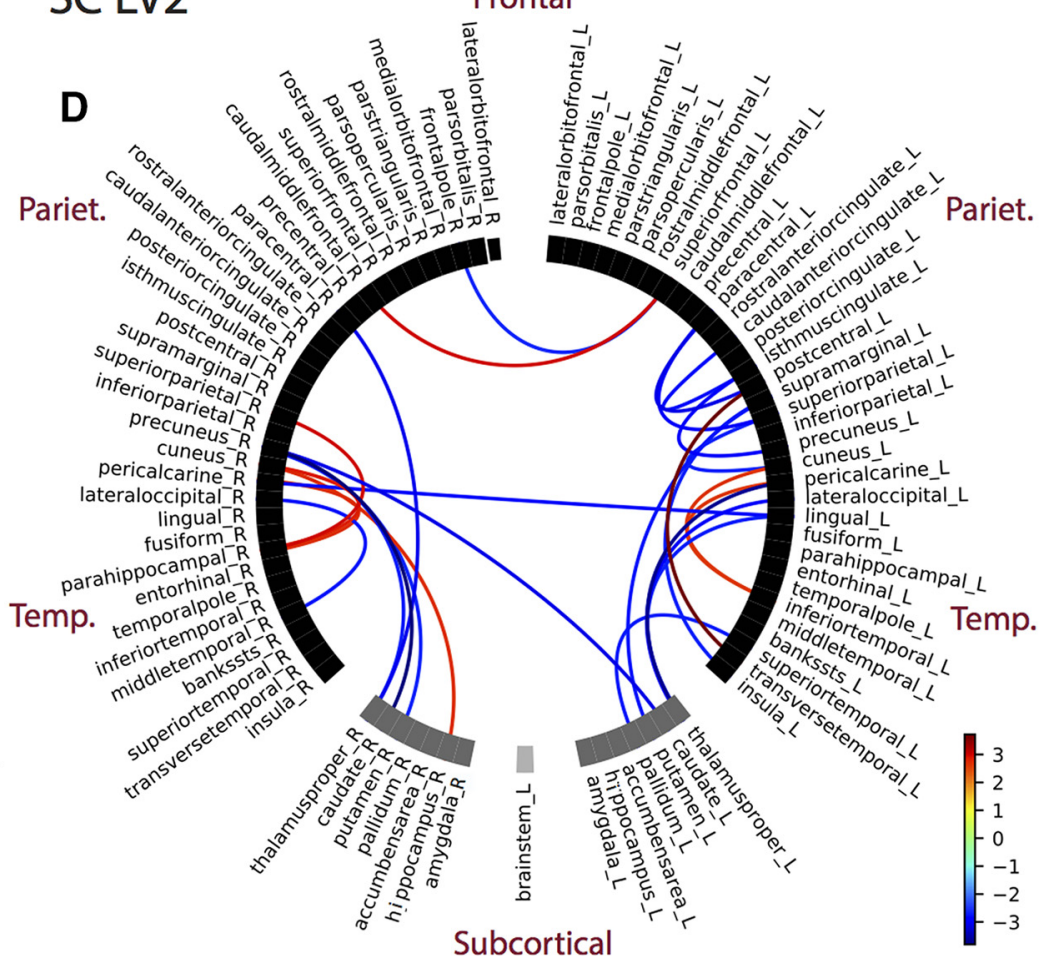

Figure 3. SC-cognition. A, LV1 correlations between SC and cognition, with Cls from bootstrap resampling. B, LV1 bootstrap ratios. These are connection loadings on the SC- cognition relationship: bootstrap threshold $=-2.5$ to 2.5 . Connections with positive bootstrap ratios contribute positively to the $S C-$ cognition correlation, and negative bootstrap ratios contribute negatively to the SC - cognition correlation. Right and left hemisphere regions, and subcortical and cortical regions, are separated by a space in the circular plot. C, LV2 correlations between SC and cognition. $\boldsymbol{D}$, LV2 bootstrap ratios. 


\section{FCLV1}

A

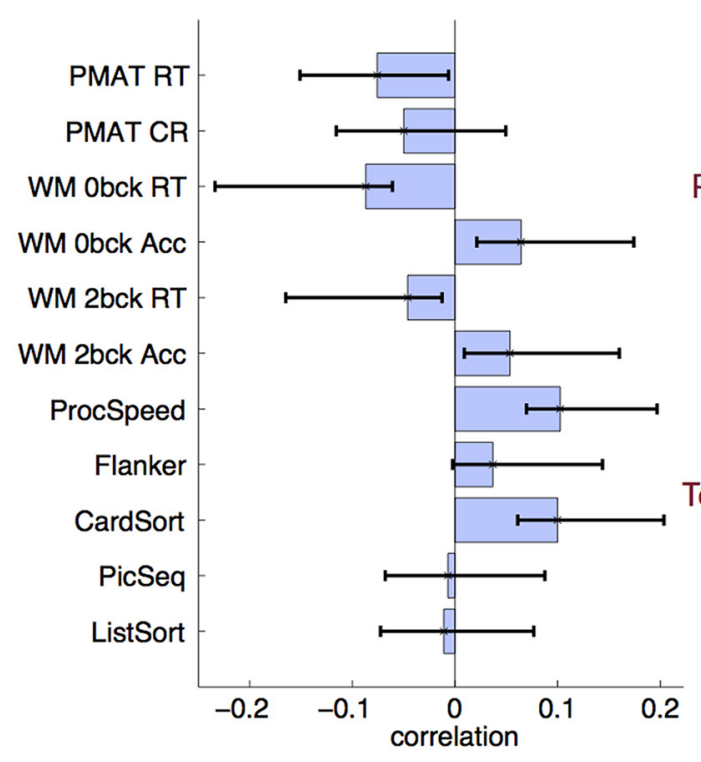

B
Frontal

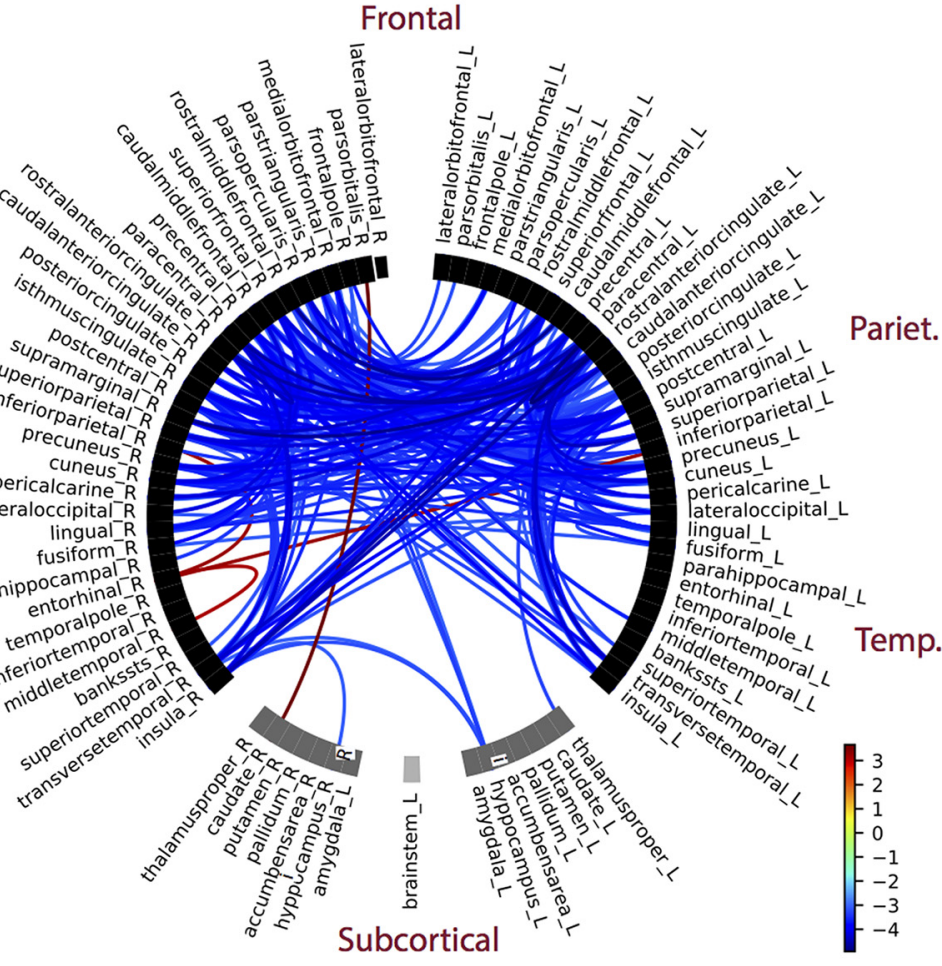

FC LV2 Frontal

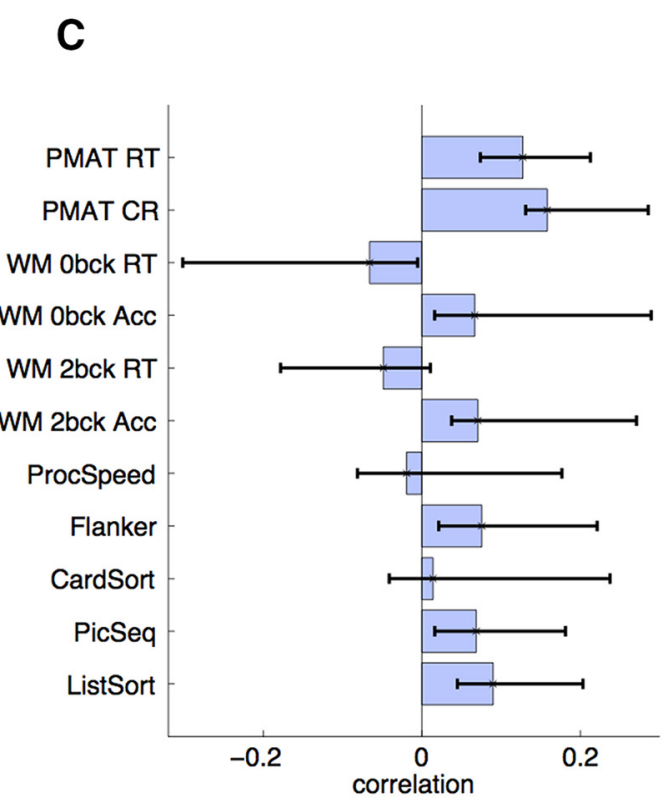

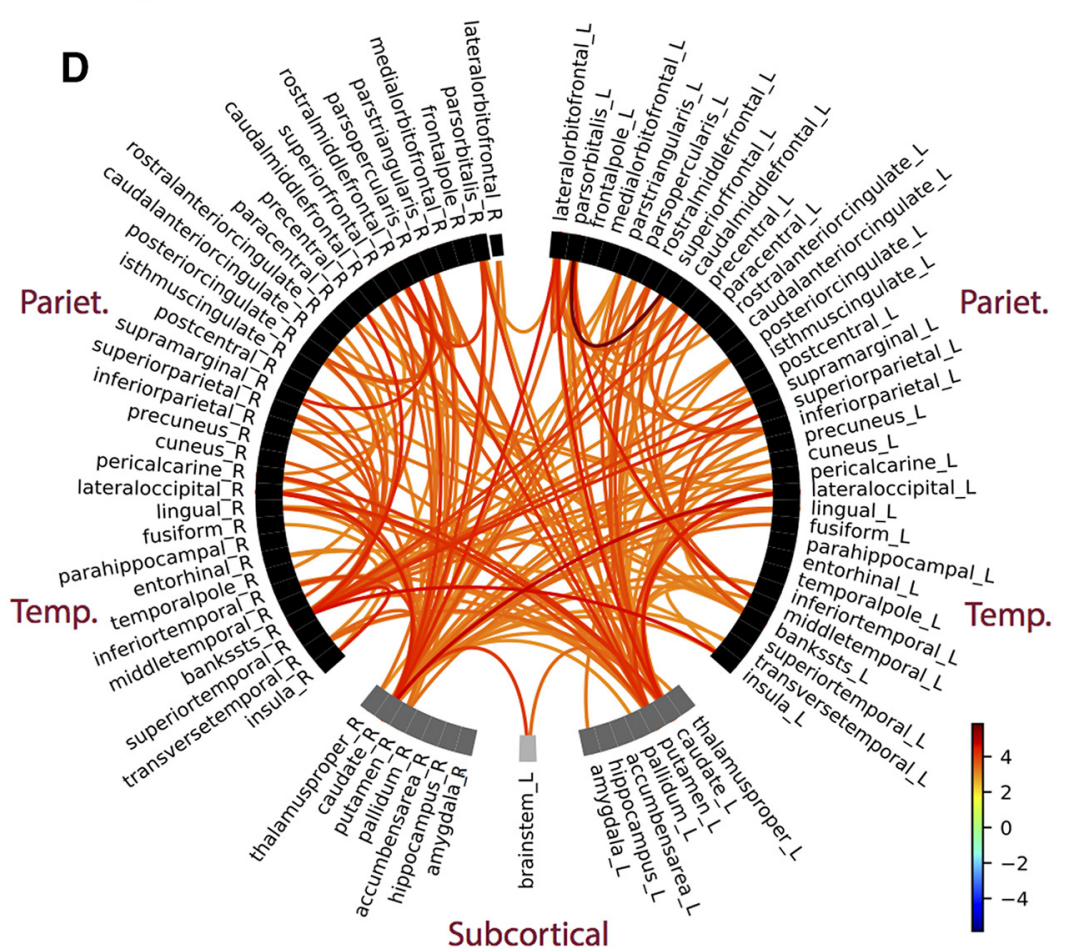

Figure 4. FC- cognition. $A$, LV1 correlations between FC and cognition, with Cls from bootstrap resampling. B, LV1 bootstrap ratios. These are connection loadings on the FC- cognition relationship: bootstrap threshold $=-3$ to 3 . Connections with high bootstrap ratios contribute positively to the $\mathrm{FC}-$ cognition correlation, and low bootstrap ratios contribute negatively to the FC- cognition correlation. Right and left hemisphere regions, and subcortical and cortical regions, are separated by a space in the circular plot. C, LV2 correlations between FC and cognition. $\boldsymbol{D}$, LV2 bootstrap ratios.

product of the PLS cognitive scores $(V)$ from the FC-cognition SVD and the cognitive scores from the SC-cognition SVD (Table 3). The behavioral patterns show some overlap across modalities. In Table 3, we also showed the correlation of individual subject scores on SC-cognition and FC-cognition LVs to examine whether highly performing subjects identified via the two analyses were comparable. To map which cognitive PCs were expressed in which LVs, we calculated the dot product between 
Table 2. Dot product between brain scores for FC- cognition and SC- cognition across both LVs

\begin{tabular}{llr}
\hline & \multicolumn{2}{l}{ FC- cognition } \\
\cline { 2 - 3 } SC- cognition & LV1 & \multicolumn{1}{c}{ LV2 } \\
\hline LV1 & 0.0043 & 0.0792 \\
LV2 & 0.0159 & -0.0419 \\
\hline
\end{tabular}

Table 3. Dot product between behavioral scores for FC- cognition and SC- cognition across both LVs

\begin{tabular}{llr}
\hline & $\mathrm{FC}-$ cognition \\
\cline { 2 - 3 } SC- cognition & $\mathrm{LV} 1$ & $\mathrm{LV} 2$ \\
\hline LV1 & $0.51(0.69, p<0.001)$ & $0.83(0.92, p<0.001)$ \\
LV2 & $0.38(0.58, p<0.001)$ & $-0.07(-0.05, p=0.25)$ \\
\hline
\end{tabular}

Dot product between behavioral scores for $\mathrm{FC}-$ cognition and SC- cognition across both LVs are shown in regular font; the correlation between behavioral individual subject scores are shown in italics.

Table 4. Dot product between cognitive PCs loadings and SC- cognition and FC- cognition LVcorrs

\begin{tabular}{lccccr}
\hline & \multicolumn{2}{l}{ SC- cognition } & & \multicolumn{2}{c}{ FC- cognition } \\
\cline { 2 - 3 } \cline { 6 - 6 } Cognition & LV1 & LV2 & & LV1 & \multicolumn{1}{c}{ LV2 } \\
\hline PC1 & 0.98 & 0.60 & & 0.59 & 0.55 \\
PC2 & -0.13 & -0.44 & & -0.83 & 0.38 \\
PC3 & -0.45 & -0.20 & & -0.02 & -0.52 \\
\hline
\end{tabular}

LVcorrs, latent variable correlations.

cognitive PC loadings and the SC-cognition and FC-cognition correlations on each LV (Table 4). We found that some cognitive PCs were particularly strongly captured in specific connectivitycognition LVs. For example, the first cognitive PC was expressed very strongly in the first SC-cognition LV. Yet, even the second SC-cognition LV mapped well onto this PC.

\section{Discussion}

In the present study, we compared whole-brain cortical and subcortical SC and rsFC from 609 subjects from the Human Connectome Project with 11 measures, including working memory (WM N-back, ListSort), executive function/cognitive flexibility (CardSort), processing speed (ProcSpeed), fluid intelligence (PMAT), episodic memory (PicSeq), and attention/inhibitory control (Flanker). The cognitive measures mapped onto three PCs, reflecting the heterogeneous nature of functions measured via the tests. PC1 was a global cognition component. PC2 expressed fluid intelligence (PMAT). PC3 emphasized a deliberate, slower, executive function/cognitive flexibility and attention/inhibitory control. We used PLS to characterize connectivity-cognition relationships, a method that is comparable to Canonical Correlation Analysis (CCA) and is increasingly used for neuroimaging data (Grady et al., 2010; Shen et al., 2015; Mišić et al., 2016; Zimmermann et al., 2018b). While either method may outperform the other in certain situations, PLS may be better suited to handling sparse data (i.e., data with many zero entries, such as our SC dataset) than sparse CCA (Grellmann et al., 2015). Although there are several methods that can be used to characterize SC/FC (Feng et al., 2015; Yu et al., 2017), PLS has been used extensively for analyzing both SC and FC (the latter generally being more dense; Grady et al., 2010; Shen et al., 2015; Mišić et al., 2016).

The conclusions of the study are twofold, as follows: (1) that unique sets of connections map onto cognitive function; and (2) that SC and FC each capture independent and unique connections related to cognition.

\section{Unique sets of connections map onto specific components of cognitive functions}

First, we showed that SC and rsFC each capture multiple sets of connection-cognition associations. Two LVs for SC and FC, respectively, each expressed a distinct and unique set of brain connections. This is in line with the view that distinct sets of connections are required to support cognition, implying more specialized connectivity (Rosenberg et al., 2013) rather than a global cognitive factor encompassed in a single set of connections (Malpas et al., 2016; Smith, 2016). We showed that while brain patterns that expressed cognitive variability did not overlap in SC and FC, the behavioral patterns did. This suggests that while different connections are related to behavior in the two modalities, the cognitive patterns mapped within these LVs overlap.

The two SC-cognition LVs each captured a limited, unique set of primarily intrahemispheric cortical and subcortical connections. SC-cognition LV1 mapped almost perfectly onto the first global cognition PC, which can be attributed to low variability across SCs. SC-cognition LV2 strongly expressed fluid intelligence (PMAT) via a set of distributed cortical and subcortical connections.

The two FC-cognition LVs each captured a large set of unique interhemispheric and intrahemispheric connections. The connectivity-cognition correlations we found were comparable to those identified previously with the HCP dataset (Hearne et al., 2016). FC-cognition LV1 expressed frontoparietal, visual, and cingulate cortical connections, as well as transverse temporal, insula, and L hippocampus connections to the rest of the cortex. rsFC in these regions was negatively associated with processing speed, executive function/cognitive flexibility, and working memory performance. We note that negative FC-cognition associations can reflect both reduced positive associations and/or altered anticorrelations (Kelly et al., 2008; Hearne et al., 2016; Spreng et al., 2016). However, anticorrelations composed a small portion of our connectomes (9.3\% of connections across all subjects), and stronger anticorrelations composed only $8.6 \%$ of connections contributing to the negative $\mathrm{FC}$-cognition associations on LV1. The second FC-cognition LV was expressed via widely distributed corticocortical and corticosubcortical connections, with strong contributions from the bilateral caudate and putamen connections to cortical regions. A large number of interhemispheric connections were expressed in this LV. The PMAT fluid intelligence measures were particularly dominant, with higher rsFC correlating with higher PMAT accuracy and reaction times, so that subjects with higher rsFC were more accurate, but slower. We note that for both SC and FC, the PMAT measures were expressed in the second LVs, which is consistent with previous findings of a fluid intelligence-connectome link (Finn et al., 2015; Hearne et al., 2016; Smith, 2016; Ferguson et al., 2017).

The patterns of connectivity that were correlated with cognitive variability in our study were consistent with those observed previously. In SC, the limited, widespread connections that associated with cognition were consistent with the findings of the study by Ponsoda et al. (2017), where only 36 distributed connections predicted higher-order cognition. A number of these connections mapped closely onto the SCs identified here. In rsFC, connectivity distributed across the cortex likely supports cognitive function (Ferguson et al., 2017). Positive rsFC-cognition associations have typically been identified in frontoparietal regions (Hearne et al., 2016), and negative associations in the default mode and dorsal attention network, including visual and cingulate-parietal connectivity (Song et al., 2008, 2009; Pamplona et al., 2015; Santarnecchi et al., 2015). FC-cognition LV1 
and LV2 captured these negative and positive associations, respectively. FC-cognition LV2 expressed several corticosubcortical connections, primarily among the putamen, caudate, thalamus, and the rest of the cortex. This is not surprising, as the cortical control of behavior is mediated via several corticostriatal-thalamic-cortical circuits (Alexander et al., 1986; Peters et al., 2016) that can be identified via structural and functional imaging (Seeley et al., 2007; Metzger et al., 2010). Connectivity from subcortical areas including the striatum have previously been tied to individual differences in phenotype and behavior (Vaidya and Gordon, 2013). The corticostriatal-thalamic cognitive control loop also includes the brainstem (Peters et al., 2016), which exhibited cognition-related connectivity with the caudate in LV2. Moreover, the striatum (putamen and caudate) is specifically involved in learning, storing, and processing memories (Packard and Knowlton, 2002), and higher WM performance has been tied to higher connectivity between the cingulo-opercular network and the putamen rsFC (Tu et al., 2012). In LV1, the only subcortical region that had negative rsFC associations with cognition was the L hippocampus, which is consistent with prior work (Salami et al., 2014). Insular connectivity, primarily with anterior cingulate regions, was also particularly prominent in LV1, a region that may be important for reactive attentional control (Jiang et al., 2015).

Interestingly, in a previous HCP study, Hearne et al. (2016) identified only positive network-level associations between rsFC and PMAT. As the authors themselves note, this may be because their network-level approach overlooks any existing edge-level connectivity-cognition relationships (Song et al., 2008, 2009; Pamplona et al., 2015; Santarnecchi et al., 2015). We replicated the positive association between rsFC and PMAT found previously (Hearne et al., 2016) within FC-cognition LV2, where fluid intelligence was emphasized as the strongest cognitive correlate.

One notable difference between SC and FC associations with cognition was that SC (LV1) correlated positively across all cognitive domains. This was not the case for FC. These results suggest that there is a global pattern of SC that supports cognition in general, akin to the global FC-behavioral mode described by Smith (2016).

\section{SC and FC each capture independent and unique connections that relate to cognition}

Second, we showed that SC and FC each captured independent and complementary features of the connectome linked to cognitive function. The connections that expressed the SC-cognition association did not overlap with behaviorally relevant connections in FC, as evidenced by the comparison of the spatial pattern of brain scores between the two analyses. While a similar suggestion has previously been made for SC versus task FC (Duda et al., 2010) and rsFC in select pathways (Hirsiger et al., 2016), it has not as of yet been examined in whole-brain SC-rsFC in as large a sample as ours. We found that far fewer connections within SC compared with FC associated with cognition, even when correcting for the sparsity of SC. This was likely due to lower variance in SC across subjects. Yet, the amount of covariance accounted for by SC and FC toward cognition was comparable, suggesting that both modalities are equally important for understanding individual cognitive differences. Behaviorally, the highly performing subjects identified by SC-cognition were the same as those identified by FC-cognition, particularly for LVs that expressed a strong association on behavioral scores.

It is important to consider that the imperfect association between SC and FC (Koch et al., 2002; Skudlarski et al., 2008; Honey et al., 2009) may impose limitations on the amount of overlapping information that can be provided by the two modalities. While SC and FC do overlap (Khalsa et al., 2014; Huang and Ding, 2016; Meier et al., 2016; Mišić et al., 2016), even within the present HCP dataset the fit between individual SC and FC is limited (Zimmermann et al., 2018a). This is in line with the present findings, whereby individual variability in the spatial connectivity patterns of SC and FC does not vary consistently across the two modalities. Limitations on the congruency between the two modalities may be exaggerated because FC is static while SC is dynamic (Park and Friston, 2013). In this vein, an investigation into functional connectivity dynamics may help to describe how the spatial contributions of SC and rsFC to cognition fluctuate over time. This is, however, beyond the scope of this study.

\section{References}

Alexander GE, DeLong MR, Strick PL (1986) Parallel organization of functionally segregated circuits linking basal ganglia and cortex. Annu Rev Neurosci 9:357-381. CrossRef Medline

Andersson JL, Sotiropoulos SN (2015) Non-parametric representation and prediction of single- and multi-shell diffusion-weighted MRI data using gaussian processes. Neuroimage 122:166-176. CrossRef Medline

Andersson JL, Skare S, Ashburner J (2003) How to correct susceptibility distortions in spin-echo echo-planar images: application to diffusion tensor imaging. Neuroimage 20:870-888. CrossRef Medline

Baldassarre A, Lewis CM, Committeri G, Snyder AZ, Romani GL, Corbetta M (2012) Individual variability in functional connectivity predicts performance of a perceptual task. Proc Natl Acad Sci U S A 109:3516-3521. CrossRef Medline

Benjamini Y, Krieger AM, Yekutieli D (2006) Adaptive linear step-up procedures that control the false discovery rate. Biometrika 93:491-507. CrossRef

Daducci A, Gerhard S, Griffa A, Lemkaddem A, Cammoun L, Gigandet X, Meuli R, Hagmann P, Thiran JP (2012) The connectome mapper: an open-source processing pipeline to map connectomes with MRI. PLoS One 7:e48121. CrossRef Medline

Duda JT, McMillan C, Grossman M, Gee JC (2010) Relating structural and functional connectivity to performance in a communication task. Med Image Comput Comput Assist Interv 13:282-289. Medline

Feinberg DA, Moeller S, Smith SM, Auerbach E, Ramanna S, Gunther M, Glasser MF, Miller KL, Ugurbil K, Yacoub E (2010) Multiplexed echo planar imaging for sub-second whole brain FMRI and fast diffusion imaging. PLoS One 5:e15710. CrossRef Medline

Feng Q, Hannig J, Marron JS (2015) Non-iterative joint and individual variation explained. arXiv:1512.04060.

Ferguson MA, Anderson JS, Spreng RN (2017) Fluid and flexible minds: intelligence reflects synchrony in the brain's intrinsic network architecture. Netw Neurosci 1:192-207. CrossRef Medline

Finn ES, Shen X, Scheinost D, Rosenberg MD, Huang J, Chun MM, Papademetris X, Constable RT (2015) Functional connectome fingerprinting: identifying individuals using patterns of brain connectivity. Nat Neurosci 18:1664-1671. CrossRef Medline

Fischl B (2012) FreeSurfer. Neuroimage 62:774-781. CrossRef Medline

Fornito A, Zalesky A, Bullmore ET (2016) Fundamentals of brain network analysis. Amsterdam, Boston: Elsevier/Academic.

Garyfallidis E (2012) Towards an Accurate Brain Tractography. Unpublished manuscript, University of Cambridge.

Garyfallidis E, Brett M, Amirbekian B, Rokem A, van der Walt S, Descoteaux M, Nimmo-Smith I and Dipy Contributors (2014) DIPY, a library for the analysis of diffusion MRI data. Frontiers in Neuroinformatics 8:8. Medline

Glasser MF, Sotiropoulos SN, Wilson JA, Coalson TS, Fischl B, Andersson JL, Xu J, Jbabdi S, Webster M, Polimeni JR, Van Essen DC, Jenkinson M (2013) The minimal preprocessing pipelines for the Human Connectome Project. Neuroimage 80:105-124. CrossRef Medline

Grady CL, Protzner AB, Kovacevic N, Strother SC, Afshin-Pour B, Wojtowicz M, Anderson JA, Churchill N, McIntosh AR (2010) A multivariate analysis of age-related differences in default mode and task-positive networks across multiple cognitive domains. Cereb Cortex 20:1432-1447. CrossRef Medline 
Grellmann C, Bitzer S, Neumann J, Westlye LT, Andreassen OA, Villringer A, Horstmann A (2015) Comparison of variants of canonical correlation analysis and partial least squares for combined analysis of MRI and genetic data. Neuroimage 107:289-310. CrossRef Medline

Hagmann P, Cammoun L, Gigandet X, Meuli R, Honey CJ, Wedeen VJ, Sporns O (2008) Mapping the structural core of human cerebral cortex. PLoS Biol 6:e159. CrossRef Medline

Hearne LJ, Mattingley JB, Cocchi L (2016) Functional brain networks related to individual differences in human intelligence at rest. Sci Rep 6:32328. CrossRef Medline

Hirsiger S, Koppelmans V, Mérillat S, Liem F, Erdeniz B, Seidler RD, Jäncke L (2016) Structural and functional connectivity in healthy aging: associations for cognition and motor behavior. Hum Brain Mapp 37:855-867. CrossRef Medline

Honey CJ, Sporns O, Cammoun L, Gigandet X, Thiran JP, Meuli R, Hagmann P (2009) Predicting human resting-state functional connectivity from structural connectivity. Proc Natl Acad Sci U S A 106:2035-2040. CrossRef Medline

Huang H, Ding M (2016) Linking functional connectivity and structural connectivity quantitatively: a comparison of methods. Brain Connect 6:99-108. CrossRef Medline

Jiang J, Beck J, Heller K, Egner T (2015) An insula-frontostriatal network mediates flexible cognitive control by adaptively predicting changing control demands. Nat Commun 6:8165. CrossRef Medline

Kelly AM, Uddin LQ, Biswal BB, Castellanos FX, Milham MP (2008) Competition between functional brain networks mediates behavioral variability. Neuroimage 39:527-537. CrossRef Medline

Khalsa S, Mayhew SD, Chechlacz M, Bagary M, Bagshaw AP (2014) The structural and functional connectivity of the posterior cingulate cortex: comparison between deterministic and probabilistic tractography for the investigation of structure-function relationships. Neuroimage 102:118127. CrossRef Medline

Klein E, Suchan J, Moeller K, Karnath HO, Knops A, Wood G, Nuerk HC, Willmes K (2016) Considering structural connectivity in the triple code model of numerical cognition: differential connectivity for magnitude processing and arithmetic facts. Brain Struct Funct 221:979-995. CrossRef Medline

Koch MA, Norris DG, Hund-Georgiadis M (2002) An investigation of functional and anatomical connectivity using magnetic resonance imaging. Neuroimage 16:241-250. CrossRef Medline

Krishnan A, Williams LJ, McIntosh AR, Abdi H (2011) Partial least squares (PLS) methods for neuroimaging: a tutorial and review. Neuroimage 56: 455-475. CrossRef Medline

Malpas CB, Genc S, Saling MM, Velakoulis D, Desmond PM, O’Brien TJ (2016) MRI correlates of general intelligence in neurotypical adults. J Clin Neurosci 24:128-134. CrossRef Medline

Matejko AA, Price GR, Mazzocco MM, Ansari D (2013) Individual differences in left parietal white matter predict math scores on the preliminary scholastic aptitude test. Neuroimage 66:604-610. CrossRef Medline

McIntosh AR, Lobaugh NJ (2004) Partial least squares analysis of neuroimaging data: applications and advances. Neuroimage 23 [Suppl 1]:S250S263. CrossRef Medline

Meier J, Tewarie P, Hillebrand A, Douw L, van Dijk BW, Stufflebeam SM, Van Mieghem P (2016) A mapping between structural and functional brain networks. Brain Connect 6:298-311. CrossRef Medline

Mennes M, Kelly C, Zuo XN, Di Martino A, Biswal BB, Castellanos FX, Milham MP (2010) Inter-individual differences in resting-state functional connectivity predict task-induced BOLD activity. Neuroimage 50: 1690-1701. CrossRef Medline

Metzger CD, Eckert U, Steiner J, Sartorius A, Buchmann JE, Stadler J, Tempelmann C, Speck O, Bogerts B, Abler B, Walter M (2010) High field FMRI reveals thalamocortical integration of segregated cognitive and emotional processing in mediodorsal and intralaminar thalamic nuclei. Front Neuroanat 4:138. CrossRef Medline

Mišić B, Betzel RF, de Reus MA, van den Heuvel MP, Berman MG, McIntosh AR, Sporns O (2016) Network-level structure-function relationships in human neocortex. Cereb Cortex 26:3285-3296. CrossRef Medline

Moeller K, Willmes K, Klein E (2015) A review on functional and structural brain connectivity in numerical cognition. Front Hum Neurosci 9:227. CrossRef Medline

Moeller S, Yacoub E, Olman CA, Auerbach E, Strupp J, Harel N, Uğurbil K (2010) Multiband multislice GE-EPI at 7 tesla, with 16-fold acceleration using partial parallel imaging with application to high spatial and temporal whole-brain fMRI. Magn Reson Med 63:1144-1153. CrossRef Medline

Packard MG, Knowlton BJ (2002) Learning and memory functions of the basal ganglia. Annu Rev Neurosci 25:563-593. CrossRef Medline

Pamplona GS, Santos Neto GS, Rosset SR, Rogers BP, Salmon CE (2015) Analyzing the association between functional connectivity of the brain and intellectual performance. Front Hum Neurosci 9:61. CrossRef Medline

Park HJ, Friston K (2013) Structural and functional brain networks: from connections to cognition. Science 342:1238411. CrossRef Medline

Peters SK, Dunlop K, Downar J (2016) Cortico-striatal-thalamic loop circuits of the salience network: a central pathway in psychiatric disease and treatment. Front Syst Neurosci 10:104. CrossRef Medline

Pezoulas VC, Zervakis M, Michelogiannis S, Klados MA (2017) Restingstate functional connectivity and network analysis of cerebellum with respect to crystallized IQ and gender. Front Hum Neurosci 11:189. CrossRef Medline

Ponsoda V, Martínez K, Pineda-Pardo JA, Abad FJ, Olea J, Román FJ, Barbey AK, Colom R (2017) Structural brain connectivity and cognitive ability differences: a multivariate distance matrix regression analysis. Hum Brain Mapp 38:803-816. CrossRef Medline

Rosenberg M, Noonan S, DeGutis J, Esterman M (2013) Sustaining visual attention in the face of distraction: a novel gradual-onset continuous performance task. Atten Percept Psychophys 75:426-439. CrossRef Medline

Salami A, Pudas S, Nyberg L (2014) Elevated hippocampal resting-state connectivity underlies deficient neurocognitive function in aging. Proc Natl Acad Sci U S A 111:17654-17659. CrossRef Medline

Santarnecchi E, Tatti E, Rossi S, Serino V, Rossi A (2015) Intelligencerelated differences in the asymmetry of spontaneous cerebral activity. Hum Brain Mapp 36:3586-3602. CrossRef Medline

Seeley WW, Menon V, Schatzberg AF, Keller J, Glover GH, Kenna H, Reiss AL, Greicius MD (2007) Dissociable intrinsic connectivity networks for salience processing and executive control. J Neurosci 27:2349-2356. CrossRef Medline

Shen K, Mišić B, Cipollini BN, Bezgin G, Buschkuehl M, Hutchison RM, Jaeggi SM, Kross E, Peltier SJ, Everling S, Jonides J, McIntosh AR, Berman MG (2015) Stable long-range interhemispheric coordination is supported by direct anatomical projections. Proc Natl Acad Sci U S A 112: 6473-6478. CrossRef Medline

Skudlarski P, Jagannathan K, Calhoun VD, Hampson M, Skudlarska BA, Pearlson G (2008) Measuring brain connectivity: diffusion tensor imaging validates resting state temporal correlations. Neuroimage 43:554561. CrossRef Medline

Smith S (2016) Linking cognition to brain connectivity. Nat Neurosci 19: 7-9. CrossRef Medline

Smith SM, Beckmann CF, Andersson J, Auerbach EJ, Bijsterbosch J, Douaud G, Duff E, Feinberg DA, Griffanti L, Harms MP, Kelly M, Laumann T, Miller KL, Moeller S, Petersen S, Power J, Salimi-Khorshidi G, Snyder AZ, Vu AT, Woolrich MW, et al (2013) Resting-state fMRI in the Human Connectome Project. Neuroimage 80:144-168. CrossRef Medline

Smith SM, Nichols TE, Vidaurre D, Winkler AM, Behrens TE, Glasser MF, Ugurbil K, Barch DM, Van Essen DC, Miller KL (2015) A positivenegative mode of population covariation links brain connectivity, demographics and behavior. Nat Neurosci 18:1565-1567. CrossRef Medline

Song M, Zhou Y, Li J, Liu Y, Tian L, Yu C, Jiang T (2008) Brain spontaneous functional connectivity and intelligence. Neuroimage 41:1168-1176. CrossRef Medline

Song M, Liu Y, Zhou Y, Wang K, Yu C, Jiang T (2009) Default network and intelligence difference. Conf Proc IEEE Eng Med Biol Soc 2009:22122215. CrossRef Medline

Sotiropoulos SN, Jbabdi S, Xu J, Andersson JL, Moeller S, Auerbach EJ, Glasser MF, Hernandez M, Sapiro G, Jenkinson M, Feinberg DA, Yacoub E, Lenglet C, Van Essen DC, Ugurbil K, Behrens TE (2013) Advances in diffusion MRI acquisition and processing in the Human Connectome Project. Neuroimage 80:125-143. CrossRef Medline

Spreng RN, Stevens WD, Viviano JD, Schacter DL (2016) Attenuated anticorrelation between the default and dorsal attention networks with aging: evidence from task and rest. Neurobiol Aging 45:149-160. CrossRef Medline

Stevens WD, Spreng RN (2014) Resting-state functional connectivity MRI 
reveals active processes central to cognition. Wiley Interdiscip Rev Cogn Sci 5:233-245. CrossRef Medline

Tu PC, Hsieh JC, Li CT, Bai YM, Su TP (2012) Cortico-striatal disconnection within the cingulo-opercular network in schizophrenia revealed by intrinsic functional connectivity analysis: a resting fMRI study. Neuroimage 59:238-247. CrossRef Medline

Uğurbil K, Xu J, Auerbach EJ, Moeller S, Vu AT, Duarte-Carvajalino JM, Lenglet C, Wu X, Schmitter S, Van de Moortele PF, Strupp J, Sapiro G, De Martino F, Wang D, Harel N, Garwood M, Chen L, Feinberg DA, Smith SM, Miller KL, et al (2013) Pushing spatial and temporal resolution for functional and diffusion MRI in the Human Connectome Project. Neuroimage 80:80-104. CrossRef Medline

Vaidya CJ, Gordon EM (2013) Phenotypic variability in resting-state functional connectivity: current status. Brain Connect 3:99-120. CrossRef Medline

Van Essen DC, Smith SM, Barch DM, Behrens TE, Yacoub E, Ugurbil K (2013) The WU-minn Human Connectome Project: an overview. Neuroimage 80:62-79. CrossRef Medline
Willmes K, Moeller K, Klein E (2014) Where numbers meet words: a common ventral network for semantic classification. Scand J Psychol 55:202211. CrossRef Medline

Yu Q, Risk BB, Zhang K, Marron JS (2017) JIVE integration of imaging and behavioral data. Neuroimage 152:38-49. CrossRef Medline

Zimmermann J, Ritter P, Shen K, Rothmeier S, Schirner M, McIntosh AR (2016) Structural architecture supports functional organization in the human aging brain at a regionwise and network level. Hum Brain Mapp 37:2645-2661. CrossRef Medline

Zimmermann J, Griffiths J, Schirner M, Ritter P, McIntosh AR (2018a) Subject-specificity of the correlation between large-scale structural and functional connectivity. Netw Neurosci. Advance online publication. Retrieved September 19, 2018. doi:10.1162/netn_a 00055.

Zimmermann J, Perry, Breakspear, Schirner, Sachdev, Wen, Kochan, Mapstone, Ritter, McIntosh, Solodkin (2018b) Differentiation of Alzheimer's disease based on local and global parameters in personalized virtual brain models. Neuroimage Clin 19:240-251. CrossRef Medline 